\title{
Editorial: Retrolental fibroplasia now retinopathy of prematurity
}

In the following pages there appears a new clinical classification for retrolental fibroplasia which has been prepared by a committee of international authorities under the chairmanship of Professor Alec Garner, of London.

The committee recommends the disuse of the original neologism, retrolental fibroplasia, coined by Messenger in 1944 and first applied by Terry in 1945, since it describes only the final stages of the disease which, being merely a non-specific scarring behind the lens, does not distinguish the condition, except by established usage, from other types of leucocoria. As the disease in its classified form originates after birth through injury to growing retinal vessels, the alternative name first introduced in 1950-1 by Parker Heath - retinopathy of prematurity - is more apt. The universal adoption of this nomenclature may take time for the acronym RLF is possibly more exclusive in medical literature than ROP and is firmly ingrained in the world literature.

The committee's classification is especially timely because of the recent resurgence of ROP in parallel with advances in the quality of neonatal care which has resulted in the survival of premature infants of very low birth weight who are at the greatest risk of developing the disease, particularly at birth weights of less than $1 \mathrm{~kg}$. This reappearance of ROP is being widely reported in published and unpublished communications, but particularly in the United States of America, where it has been estimated that the annual number of infants blinded in 1979 was 546, a figure comparable to that recorded during the peak years of the original epidemic of 1943-53.

Indeed this 'second epidemic' as it has been called has begun to cast doubt on the role of oxygen as the important aetiological factor, since many cases of ROP are now occurring despite meticulous control of oxygen administration and even where no supplemental oxygen has been administered. Some clinicians are turning to re-examine the possible role of the many other abnormal factors directly, indirectly, or even remotely associated with prematurity, seeming to retreat to the despairing days of the first epidemic when little or nothing was known about the selective and injurious action of oxygen upon growing retinal vessels. Much clinical, patho- logical, and experimental research was carried out to prove that fact, and the subsequent control of oxygen therapy, albeit with the high price paid in morbidity and mortality, led to a dramatic fall in the incidence of ROP and established without question the causative role of the overuse of oxygen. Is it now to be supposed that oxygen is injurious in only the later stages of prematurity and not in the earlier stages, despite experimental evidence that the severity of the oxygen effect is directly proportional to the degree of immaturity of the retinal vessels? It would seem more reasonable to conceive that oxygen is injurious throughout the whole period of retinal vascular growth, the normal percentage in air being a sufficiently foreign environment in the most vulnerable infants. In utero the oxygen saturation in the arterial blood is approximately $50 \%$; after birth it rises to approximately $90 \%$ saturation without the addition of oxygen to room air.

On the other hand the exact biochemical events which lead to oxygen vasoobliteration are unknown and may well be set in train by something other than oxygen. Experimentally it has been shown that growing retinal vessels may be closed by other agents, while the vasoproliferative phase may also occur in experimental situations where oxygen can certainly be excluded. So, despite the many current speculations which seem feasible to some and not to others, no certain opinions can at present be expressed as to the cause or causes of the second epidemic of ROP.

A renewed and vigorous research effort is urgently required. Pathologically we particularly need to know exactly what is happening to the retinal vessels in these tiny babies. Experimentally further work on the biochemical elucidation of oxygen vasoobliteration and vasoproliferation is required, especially in search of antagonists. And clinically there is a need to collect accurate incidence data, which have so far lacked a universally accepted classification, to provide criteria for estimating the effectiveness of preventive and therapeutic agents. This international classification is therefore especially to be both welcomed and recommended as an important step in meeting this renewed challenge of an old enemy. 